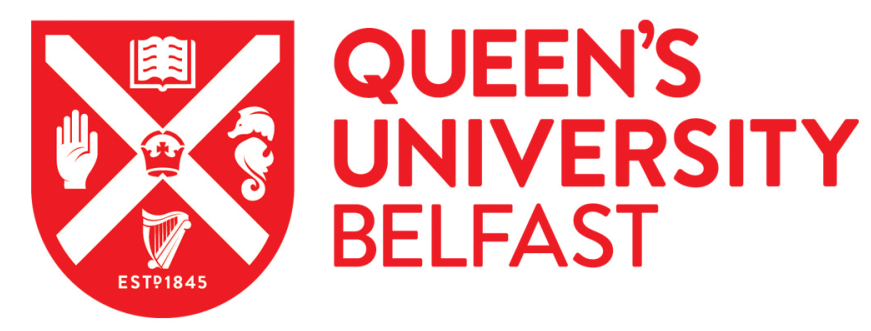

\title{
Switched Diversity Techniques for Indoor Off-body Communications Channels: An Experimental Analysis and Modeling
}

Yoo, S. K., Cotton, S. L., \& Scanlon, W. G. (2016). Switched Diversity Techniques for Indoor Off-body Communications Channels: An Experimental Analysis and Modeling. IEEE Transactions on Antennas and Propagation, 64(7), 3201-3206. https://doi.org/10.1109/TAP.2016.2557851

Published in:

IEEE Transactions on Antennas and Propagation

Document Version:

Publisher's PDF, also known as Version of record

Queen's University Belfast - Research Portal:

Link to publication record in Queen's University Belfast Research Portal

Publisher rights

Copyright 2016 the authors.

This is an open access article published under a Creative Commons Attribution License (https://creativecommons.org/licenses/by/4.0/), which permits unrestricted use, distribution and reproduction in any medium, provided the author and source are cited.

\section{General rights}

Copyright for the publications made accessible via the Queen's University Belfast Research Portal is retained by the author(s) and / or other copyright owners and it is a condition of accessing these publications that users recognise and abide by the legal requirements associated with these rights.

Take down policy

The Research Portal is Queen's institutional repository that provides access to Queen's research output. Every effort has been made to ensure that content in the Research Portal does not infringe any person's rights, or applicable UK laws. If you discover content in the Research Portal that you believe breaches copyright or violates any law, please contact openaccess@qub.ac.uk. 


\section{Switched Diversity Techniques for Indoor Off-Body Communication Channels: An Experimental Analysis and Modeling}

\author{
Seong Ki Yoo, Simon L. Cotton, and William G. Scanlon
}

\begin{abstract}
This communication investigates the potential improvement in signal reliability for indoor off-body communications channels operating at $5.8 \mathrm{GHz}$ using switched diversity techniques. In particular, we investi-gate the performance of switch-and-stay combining (SSC), switch-and-examine combining (SEC), and (SECps) schemes, which utilize multiple spatially separated antennas at the base station. During the measurements, a test subject, wearing an antenna on his chest, performed a number of walking movements toward and then away from a uniform linear array. It was found that all the considered diversity schemes provided a worthwhile signal improvement. However, the performance of the diversity systems varied according to the switching threshold that was adopted. To model the fading envelope observed at the output of each of the combiners, we have applied diversity specific equations developed under the assumption of Nakagami- $m$ fading. As a measure of the goodness-of-fit, the Kullback-Leibler divergence between the empirical and theoretical probability density functions (pdfs) was calculated and found to be close to 0 . To assist with the interpretation of the goodness-of-fit achieved in this communication, the standard deviation $\sigma$ of a zero-mean, $\sigma^{2}$ variance Gaussian pdf used to approximate a zero-mean, unit variance Gaussian pdf is also presented. These were generally quite close to 1 indicating that the theoretical models provided an adequate fit to the measured data.
\end{abstract}

Index Terms - Channel measurements, Nakagami- $m$ fading, off-body communications, switched diversity.

\section{INTRODUCTION}

One well-known method of mitigating the deleterious effects of fading in wireless communication systems is to employ diversity reception techniques [1]. While there are many different approaches currently in use, which include time, frequency, and polarization techniques, it is space diversity that is the most commonly applied as there is no need to increase the transmit power or bandwidth [2]. When combining the signals received at multiple spatially separated antennas, two different categories of combining are prevalently used, namely, switched combining and gain combining [3]. In the former grouping, which includes pure selection combining (PSC) and threshold selection combining (TSC), the receiver chooses one of the available diversity paths according to a predefined criteria. In the latter grouping, the output of a combiner is a linear combination of the signals received by all the diversity paths. This category of combining techniques includes equal gain combining (EGC) and maximal ratio combining (MRC).

In general, gain combining schemes provide a better performance than switched combining schemes with MRC being recognized as the optimal combining scheme. Historically, the use of MRC introduced a tradeoff between the receiver complexity and the performance of a diversity system [4]. Nowadays, however, the complexity

Manuscript received May 1, 2015; revised March 9, 2016; accepted April 10, 2016. Date of publication April 25, 2016; date of current version July 5, 2016. This work was supported in part by the Leverhulme Trust under Grant PLP-2011-061, in part by the Engineering and Physical Sciences Research Council under Grant EP/H044191/1 and Grant EP/L026074/1, and in part by the U.K. Royal Academy of Engineering under the Research Fellowship.

The authors are with the Institute of Electronics, Communications and Information Technology, Queen's University Belfast, Belfast BT3 9DT, U.K. (e-mail: syoo02@qub.ac.uk; simon.cotton@qub.ac.uk; w.scanlon@qub.ac.uk).

Color versions of one or more of the figures in this communication are available online at http://ieeexplore.ieee.org.

Digital Object Identifier 10.1109/TAP.2016.2557851 of MRC schemes is no longer a major issue through the development of combiners such as soft-bit MRC [5]. Nonetheless, switched combining has still gained widespread use because of its low complexity and ease of implementation. In a PSC system, the combiner monitors the input signal-to-noise ratio (SNR) of all the diversity paths simultaneously and selects the branch with the highest input SNR. In contrast, a receiver employing a TSC scheme switches from one branch to another only when the input SNR of the current branch falls below the predetermined threshold [4]. This approach has the advantage that the receiver is not needlessly monitoring the input SNR of all the diversity paths and switching between branches when the input SNR of the current branch is at a level that is acceptable for supporting the desired information recovering capability.

The TSC scheme can be broadly divided into three different schemes, namely, switch-and-stay combining (SSC), switch-andexamine combining (SEC), and SEC with postexamining selection (SECps) schemes. In an SSC scheme, the receiver simply switches from one branch to another when the input SNR of the current branch drops below the predetermined threshold. With an SEC scheme, the receiver switches to an alternative branch and examines its input SNR. If it is not above the predetermined threshold, the receiver switches to another alternative branch and examines the input SNR again. This process continues until the receiver either finds an acceptable branch or determines that no acceptable branch is available. In the latter case, the receiver usually selects the last branch that was examined. For both the SSC and SEC schemes, when the input SNR of the current branch falls below the switching threshold, path switching between branches always happens regardless of whether the input SNR of another branch is above or below the input SNR of the current branch. In fact, in the latter case, path switching can degrade the system performance. A receiver employing an SECps scheme works in exactly the same manner as an SEC scheme when an acceptable branch is available. However, when there is no acceptable branch available, SECps selects the branch with the highest input SNR instead of the previously examined one.

Over the last few years, a number of diversity techniques have been studied in the context of body centric communications [6]-[10]. In [6], switched diversity combining was employed in on-body communications systems. Here, an improvement in outage probability, a reduction in power consumption, and a low switching rate were achieved using cooperative diversity with an SEC scheme. However, the majority of studies on diversity techniques for off-body communications have considered only PSC, EGC, and MRC [7]-[10]. Therefore, in this communication, we investigate the possible signal reliability improvement for indoor off-body communications channels operating at $5.8 \mathrm{GHz}$ using switched diversity techniques. Since no further benefit can be obtained from having more than two signal branches in SSC [11], we consider dual-branch SSC, $L$-branch SEC, and $L$-branch SECps schemes. Furthermore, for the first time, we model the fading observed at the output of each switched diversity combiner for off-body communications systems operating in Nakagami- $m$ fading channels using diversity specific analytical equations. Since it is difficult to measure the time-varying SNR in practice, we present new analytical expressions for the output envelope instead of those for the output SNR [1].

The remainder of this communication is organized as follows. In Section II, we briefly review the characteristics of the Nakagami- $m$ fading model before introducing theoretical equations for the probability density functions (pdfs) of dual-branch SSC, $L$-branch 
SEC, and $L$-branch SECps operating in independent and identically distributed (i.i.d) Nakagami- $m$ fading channels. In Section III, the measurement setup, environment, and procedure are described. In Section IV, the power imbalance, cross correlation, and diversity gain (DG) for the considered combiners are presented in conjunction with some examples of the model fitting. Finally, we conclude this communication with a summary of the main findings in Section V.

\section{SWITCHED DIVERSITY SySTEMS OPERATING IN NAKAGAMI- $m$ FADING CHANNELS}

\section{A. Nakagami-m Fading Model}

There are a number of different models used to describe the statistical behavior of fading in mobile radio propagation channels. Among them, the Nakagami- $m$ fading model has been found to provide a good fit to body centric [12] and land-mobile propagation [13]. The pdf, $f_{\gamma}(\gamma)$, and cumulative distribution function (cdf), $F_{\gamma}(\gamma)$, of the SNR, $\gamma$, over a Nakagami- $m$ fading channel can be expressed as [4]

$$
\begin{aligned}
& f_{\gamma}(\gamma)=\frac{m^{m} \gamma^{m-1}}{\Gamma(m) \bar{\gamma} m} \exp \left(-\frac{m \gamma}{\bar{\gamma}}\right) \\
& F_{\gamma}(\gamma)=1-\frac{\Gamma\left(m, \frac{m \gamma}{\bar{\gamma}}\right)}{\Gamma(m)}
\end{aligned}
$$

where $m$ is the fading severity parameter, $\bar{\gamma}=E[\gamma]$ is the average SNR with $E[\cdot]$ denoting statistical expectation, $\Gamma(\cdot)$ is gamma function, and $\Gamma(\cdot, \cdot)$ is the upper incomplete gamma function. When $m=1$, the Nakagami- $m$ fading model becomes equivalent to the Rayleigh fading model [14]. It can also be used to describe the fading conditions that are worse than those found in a Rayleigh fading environment $(0.5 \leq m<1)$ and give a good approximation of Ricean fading $(m>1)$ [14].

\section{B. Dual-Branch SSC Over Nakagami-m Fading Channels}

For dual-branch SSC systems in which the input SNR is i.i.d. at both branches, the pdf of the output SNR, $\gamma$, can be expressed as [11]

$$
f_{\mathrm{SSC}}(\gamma)= \begin{cases}F_{\gamma}\left(\gamma_{T}\right) f_{\gamma}(\gamma), & \gamma<\gamma_{T} \\ {\left[1+F_{\gamma}\left(\gamma_{T}\right)\right] f_{\gamma}(\gamma),} & \gamma \geq \gamma_{T}\end{cases}
$$

where $\gamma_{T}$ is the predetermined switching threshold, and $f_{\gamma}(\cdot)$ and $F_{\gamma}(\cdot)$ denote the pdf and cdf of the input SNR at one branch, respectively. To obtain the pdf of the output SNR for dual-branch SSC operating over i.i.d. Nakagami- $m$ fading channels, we simply substitute (1) and (2) into (3). Then, performing a simple transformation of variables using the relationship $\gamma=r^{2} \bar{\gamma} / \bar{r}^{2}[4]$ and letting $\Omega=E\left[R^{2}\right]=\bar{r}^{2}$, we can obtain the pdf of the dual-branch SSC envelope, $R$, as shown in (4) at the bottom of the page.

\section{L-Branch SEC Over Nakagami-m Fading Channels}

For $L$-branch SEC systems in which the input SNR is i.i.d. at each of the $L$ branches, the pdf of the output SNR, $\gamma$, can be expressed as [11]

$$
f_{\mathrm{SEC}}(\gamma)= \begin{cases}F_{\gamma}\left(\gamma_{T}\right)^{L-1} f_{\gamma}(\gamma), & \gamma<\gamma_{T} \\ \sum_{i=0}^{L-1}\left[F_{\gamma}\left(\gamma_{T}\right)\right]^{i} f_{\gamma}(\gamma), & \gamma \geq \gamma_{T} .\end{cases}
$$

Similarly, the pdf of the output envelope, $R$, of an $L$-branch SEC combiner can be obtained by substituting (1) and (2) into (5) and then performing the same transformation of variables used above to yield (6), as shown at the bottom of the page. It has been shown that an SEC system with two branches can provide the same performance as a dual-branch SSC scheme [11], i.e., by letting $L=2$, (6) then reduces to (4).

\section{L-Branch SECps Over Nakagami-m Fading Channels}

For $L$-branch SECps systems in which the input SNR is i.i.d. at each of the $L$ branches, the pdf of the output SNR, $\gamma$, can be expressed as [15]

$$
f_{\mathrm{SECps}}(\gamma)= \begin{cases}L\left[F_{\gamma}(\gamma)\right]^{L-1} f_{\gamma}(\gamma), & \gamma<\gamma_{T} \\ \sum_{i=0}^{L-1}\left[F_{\gamma}\left(\gamma_{T}\right)\right]^{i} f_{\gamma}(\gamma), & \gamma \geq \gamma_{T} .\end{cases}
$$

Again, the pdf of the output envelope, $R$, of an $L$-branch SECps combiner can be obtained by substituting (1) and (2) into (7) and then performing the same transformation of variables to give (8), as shown at the bottom of the following page.

\section{FIELD MEASUREMENTS}

The measurements performed in this communication were conducted at $5.8 \mathrm{GHz}$ in an indoor laboratory $(4.75 \mathrm{~m} \times 9.14 \mathrm{~m} \times$ $2.70 \mathrm{~m}$ ) as shown in Fig. 1(a). The hypothetical base station receiver consisted of four identical sleeve dipole antennas horizontally aligned along a straight line with an equal spacing of half wavelength. These four receive antennas were mounted, such that they were vertically polarized on a nonconductive height adjustable stand at an elevation of $0.83 \mathrm{~m}$ above the floor level. They were connected to ports $1,2,3$, and 4 of a Rohde \& Schwarz ZVB-8 vector network

$$
\begin{aligned}
f_{\mathrm{SSC}}(r)=\left\{\begin{array}{l}
\left(1-\frac{\Gamma\left(m, \frac{m}{\Omega} r_{T}^{2}\right)}{\Gamma(m)}\right)\left(\frac{2 m^{m} r^{2 m-1}}{\Gamma(m) \Omega^{m}} \exp \left(-\frac{m}{\Omega} r^{2}\right)\right), \quad r<r_{T} \\
\left(2-\frac{\Gamma\left(m, \frac{m}{\Omega} r_{T}^{2}\right)}{\Gamma(m)}\right)\left(\frac{2 m^{m} r^{2 m-1}}{\Gamma(m) \Omega^{m}} \exp \left(-\frac{m}{\Omega} r^{2}\right)\right), \quad r \geq r_{T}
\end{array}\right. \\
f_{\mathrm{SEC}}(r)= \begin{cases}{\left[\left(1-\frac{\Gamma\left(m, \frac{m}{\Omega} r_{T}^{2}\right)}{\Gamma(m)}\right)\right]^{L-1}\left(\frac{2 m^{m} r^{2 m-1}}{\Gamma(m) \Omega^{m}} \exp \left(-\frac{m}{\Omega} r^{2}\right)\right),} & r<r_{T} \\
\sum_{i=0}^{L-1}\left[\left(1-\frac{\Gamma\left(m, \frac{m}{\Omega} r_{T}^{2}\right)}{\Gamma(m)}\right)\right]^{i}\left(\frac{2 m^{m} r^{2 m-1}}{\Gamma(m) \Omega^{m}} \exp \left(-\frac{m}{\Omega} r^{2}\right)\right), & r \geq r_{T}\end{cases}
\end{aligned}
$$




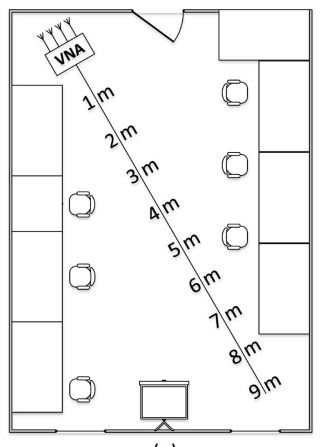

(a)

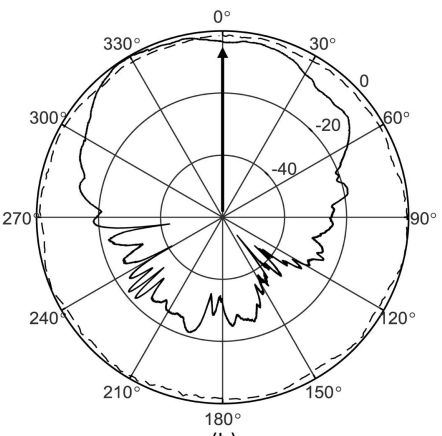

(b)
Fig. 1. (a) Indoor laboratory environment $\left(43.42 \mathrm{~m}^{2}\right)$. (b) Measured azimuthal radiation pattern for the sleeve dipole antenna in free space (dashed lines) and located on the central chest region (continuous lines) of the test subject. Black arrow in (b): direction that the test subject was facing.

analyzer using low-loss coaxial cables and configured to record the magnitude of the $b_{1}$ wave quantity incident on ports $1,2,3$, and 4 with a bandwidth of $10 \mathrm{kHz}$. The magnitude of the $b_{1}$ measurements was automatically collected and stored on a laptop through a local area network connection, providing an effective channel sampling frequency of $56 \mathrm{~Hz}$. A premeasurement calibration was performed to reduce the effects of known systematic errors and cable loss using a Rohde \& Schwarz ZV-Z51 calibration unit.

For the transmitter, an ML5805 transceiver $^{1}$ manufactured by RFMD was configured to generate a continuous wave signal with a power level of $+17.6 \mathrm{dBm}$. It was mounted in a vertically polarized orientation and parallel to the central chest region of an adult male of height $1.83 \mathrm{~m}$ and weight $80 \mathrm{~kg}$. The antennas used by both the transmitter and the hypothetical base station were omnidirectional sleeve dipole antennas with +2.3 -dBi gain (Mobile Mark model PSKN3-24/55S). ${ }^{2}$ The measured azimuthal radiation patterns for the sleeve dipole antenna in free space and placed at the central chest region are shown in Fig. 1(b). In the experiments conducted here, two different measurement scenarios were considered. These were the line of sight (LOS) and non-LOS (NLOS) walking movements, where the test subject walked toward and then away from the receiver in straight line (between 1 and $9 \mathrm{~m}$ away from the receiver). To improve the validity and robustness of the parameter estimates obtained in this communication, all the measurements were repeated five times for each of the scenarios. The mean recorded noise levels were observed to be $-98.5,-98.6,-98.6$, and $-98.5 \mathrm{dBm}$ for branches $1,2,3$, and 4 , respectively. The minimum data set sizes (from the individual trials) were 2417 and 2119 for the LOS and NLOS walking scenarios, respectively.

\footnotetext{
${ }^{1}$ http://datasheet.octopart.com/ML5805DM-Micro-Linear-datasheet8614608.pdf (11/03/2015)

${ }^{2}$ http://www.mobilemark.com/wp-content/uploads/2015/04/antenna-spec117-pskn3-2400.pdf (11/03/2015)
}

\section{RESULTS}

\section{A. Envelope Correlation and Power Imbalance}

For a diversity scheme to be effective, each branch should receive the statistically independent versions of the transmitted signal reducing the likelihood that all the branches are experiencing correlated fading. In general, two signals are said to be suitably decorrelated if their cross-correlation coefficient is $<0.7$ [1]. The cross-correlation coefficient of the fading and the power imbalance between the signal power received at each of the antennas used in this communication was calculated using the approach proposed in [16]. For brevity, we do not exhaustively list our results, but note that for all the cases, the estimated cross-correlation coefficients were always $<0.2$. In addition, the estimated power imbalance for the dual-branch configuration (branches 1 and 4) was 1.1 and $0.4 \mathrm{~dB}$ for the LOS and NLOS scenarios, respectively. For the four-branch configuration, the mean power imbalance averaged over all the possible pairs was $2.0 \mathrm{~dB}$ (LOS) and $0.7 \mathrm{~dB}$ (NLOS), while the maximum power imbalance was $3.7 \mathrm{~dB}$ (LOS) and $1.2 \mathrm{~dB}$ (NLOS). The low cross-correlation coefficients and power imbalances obtained suggest that a receiver equipped with multiple antennas should be adequately positioned to supply a worthwhile DG.

\section{B. Diversity Gain and Switching Threshold}

In this communication, we empirically evaluate the performance of the SSC, SEC, and SECps schemes for use in off-body communications in terms of their DG. This quantifies the improvement in the signal reliability of a diversity combiner over a single branch receiver and is generally expressed in decibel as

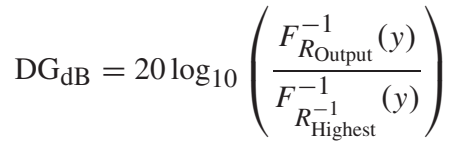

where $F_{R_{\text {Output }}}^{-1}(y)$ and $F_{R_{\text {Highest }}}^{-1}(y)$ represent the inverse transforms of the empirical cdfs of the combiner output and the branch with the highest mean at a cumulative probability of $y$, respectively. Please note that all the DG calculations were made at a cumulative probability of 0.1 ( $10 \%$ cdf level), which is equivalent to a signal reliability of $90 \%$. As an additional quantitative measure, the DGs of the SSC, SEC, and SECps schemes were compared with those calculated for a PSC.

Fig. 2 shows a short excerpt of the received signal power at branches 1 and 4 along with the output signal of the hypothetical dual-branch SSC, SEC, and SECps combiners with a switching threshold of $-66 \mathrm{dBm}$ for the LOS walking scenario during the first trial. As we can see, when branch switching occurs, the dual-branch SSC and SEC schemes operate identically, but dual-branch SECps works slightly differently. This can be explained from the way that the TSC system with dual-branch SSC and SEC schemes switches to the other branch whenever the signal level of the currently selected branch falls below the predetermined switching threshold. This path switching occurs irrespective of whether the signal level of the

$$
f_{\mathrm{SECps}}(r)= \begin{cases}L\left[\left(1-\frac{\Gamma\left(m, \frac{m}{\Omega} r^{2}\right)}{\Gamma(m)}\right)\right]^{L-1}\left(\frac{2 m^{m} r^{2 m-1}}{\Gamma(m) \Omega^{m}} \exp \left(-\frac{m}{\Omega} r^{2}\right)\right), \quad r<r_{T} \\ \sum_{i=0}^{L-1}\left[\left(1-\frac{\Gamma\left(m, \frac{m}{\Omega} r_{T}^{2}\right)}{\Gamma(m)}\right)\right]^{i}\left(\frac{2 m^{m} r^{2 m-1}}{\Gamma(m) \Omega^{m}} \exp \left(-\frac{m}{\Omega} r^{2}\right)\right), \quad r \geq r_{T}\end{cases}
$$


TABLE I

Average DGs for the Dual- and Four-Branch PSC, Dual-Branch SSC, Dual- And Four-Branch SEC, AND Dual- AND Four-Branch SECps IN THE LOS AND NLOS WALKING SCENARIOS WiTH THREE DiFFERENT SWITCHING THRESHOLDS

\begin{tabular}{|c|c|c|c|c|c|c|c|c|c|}
\hline \multirow{3}{*}{ Scenario } & \multirow{3}{*}{ Threshold (dBm) } & \multicolumn{8}{|c|}{ Diversity Gain (dB) } \\
\hline & & \multirow[b]{2}{*}{ 2-SSC } & \multirow{2}{*}{\multicolumn{2}{|c|}{$\begin{array}{c}\text { TSC } \\
\text { 2-SECps }\end{array}$}} & & \multirow[b]{2}{*}{ 4-SECps } & & \multicolumn{2}{|c|}{ PSC } \\
\hline & & & & & 4-SEC & & & 2-PSC & 4-PSC \\
\hline \multirow{3}{*}{ LOS } & -80 & -0.1 & -0.1 & -0.1 & -0.5 & -0.5 & & & \\
\hline & -60 & 1.8 & 1.8 & 4.1 & 2.7 & 5.4 & LOS & 4.2 & 5.8 \\
\hline & -40 & -0.9 & -0.9 & 4.2 & -2.2 & 5.8 & & & \\
\hline \multirow{3}{*}{ NLOS } & -80 & 1.6 & 1.6 & 1.6 & 1.5 & 1.5 & & & \\
\hline & -60 & 1.1 & 1.1 & 5.4 & 2.2 & 7.9 & NLOS & 5.4 & 7.9 \\
\hline & -40 & 0.1 & 0.1 & 5.4 & 0.1 & 7.9 & & & \\
\hline
\end{tabular}

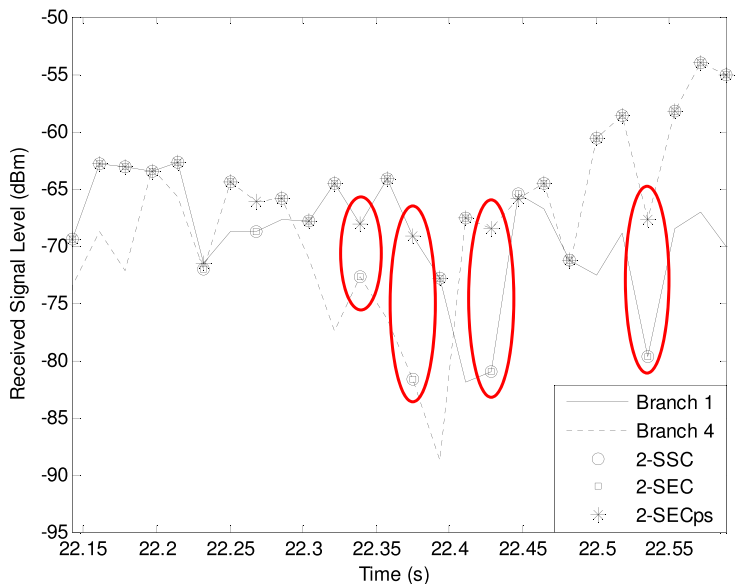

Fig. 2. Received signal power levels at branches 1 and 4 alongside the output signal power of dual-branch SSC, SEC, and SECps with a switching threshold of $-66 \mathrm{dBm}$ for the LOS walking scenario during the first trial where the red ellipses indicate the time slot in which dual-branch SECps scheme operated differently than the dual-branch SSC and SEC schemes.

other branch is above or below the switching threshold. Moreover, the signal level of the other branch can be even lower than the one for the currently selected branch. In this case, the system performance is degraded instead of improved.

Similar to the dual-branch SEC, a TSC system using a dual-branch SECps scheme examines and finds an acceptable branch, which is above the switching threshold. However, unlike SEC, after examining all the branches, the SECps scheme switches to the branch with the highest signal level instead of the last examined branch when no acceptable one is found. As an example of this behavior, consider the first two red ellipses shown in Fig. 2, here, the dual-branch SSC and SEC schemes switched from branch 1 to 4 despite the fact that branch 4 had an even lower signal level than branch 1, whereas the dual-branch SECps scheme stayed with branch 1.

Table I shows the mean DG statistics for dual-branch SSC, SEC, and SECps and four-branch SEC and SECps at 90\% signal reliability for both the LOS and NLOS walking scenarios with three different switching thresholds, namely, low $(-80 \mathrm{dBm})$, medium $(-60 \mathrm{dBm})$, and high $(-40 \mathrm{dBm})$. These values were determined based on the average received signal power levels measured at each branch, which ranged from -65.9 to $-55.0 \mathrm{dBm}$. For comparison, the mean DGs for dual- and four-branch PSC are also shown. It should be noted that branches 1 and 4 were chosen for the analysis of dual-branch PSC, SSC, SEC, and SECps.

As expected, the DGs for the dual-branch SSC were the same as the one for dual-branch SEC. Among the three different TSC schemes, the SECps scheme provided the highest DG. Also from Table I,
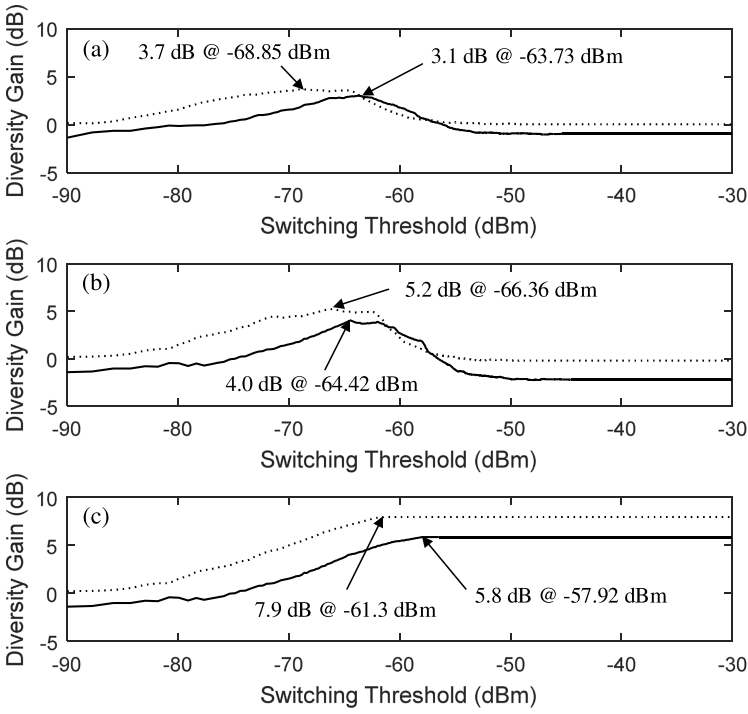

Fig. 3. Average DG of (a) dual-branch SSC, (b) four-branch SEC, and (c) four-branch SECps for increasing values of switching threshold for the LOS (continuous lines) and NLOS (dashed lines) walking scenarios along with the maximum achievable DG at the optimum switching threshold.

it is clear that the estimated DGs for both the dual- and four-branch TSC systems were different according to the switching threshold. To further investigate the effect of different switching thresholds on the achievable DG, Fig. 3 shows the average DG for dual-branch SSC, four-branch SEC, and SECps with different switching threshold values ranging between -90 and $-30 \mathrm{dBm}$ for both the LOS and NLOS walking scenarios.

As shown in Fig. 3, the performance of all the TSC schemes strongly depends on the predetermined switching threshold and there exists an optimum switching threshold that maximizes the DG. It is clear that a greater DG was achieved by all the combiners for the NLOS scenario than the LOS scenario when the optimum switching threshold was chosen. It was also observed that the DG obtained for four-branch SECps with the optimum switching threshold was the same as the one for the respective PSC system shown in Table I. However, when the switching threshold was considerably low or high compared with the average received signal power level, there was no benefit to using a TSC scheme. Interestingly, in contrast with dual-branch SSC and four-branch SEC, the DG for four-branch SECps kept the same DG beyond the optimum switching threshold. This is most likely due to the fact that the SECps scheme examines all the branches and switches to the branch with the highest signal level when there is no acceptable branch available. Therefore, when the switching threshold is high and the signal level of all diversity branches are below the predetermined switching threshold, it operates 

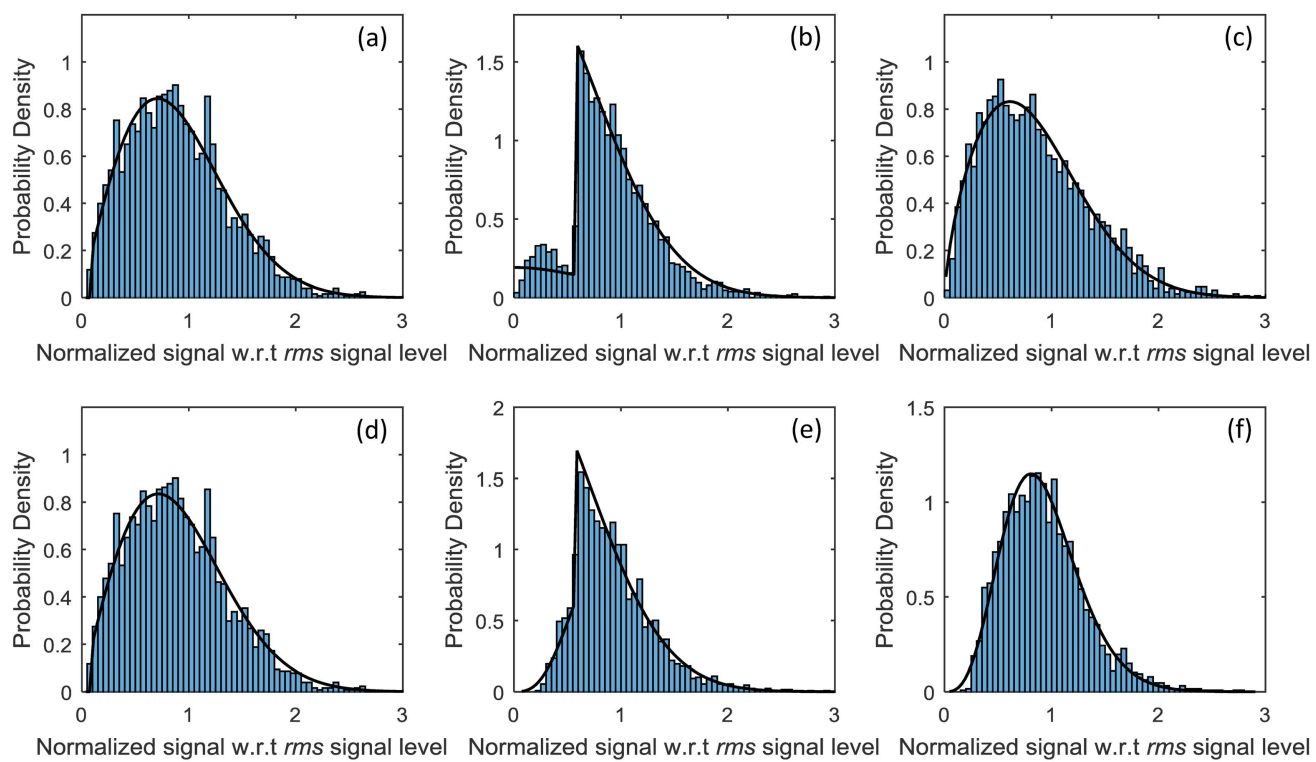

Fig. 4. Empirical (bars) and theoretical (continuous lines) pdfs for the LOS walking scenario during the second trial. (a) 4-SEC with low threshold ( $-80 \mathrm{dBm}$ ). (b) 4-SEC with medium threshold $(-60 \mathrm{dBm})$. (c) 4-SEC with high threshold $(-40 \mathrm{dBm})$. (d) 4-SECps with low threshold $(-80 \mathrm{dBm})$. (e) 4-SECps with medium threshold $(-60 \mathrm{dBm})$. (f) 4-SECps with high threshold $(-40 \mathrm{dBm})$.

TABLE II

Average Parameter Estimates ( $\bar{m}$ and $\bar{\Omega}$ ), Mean Signal Power of the Output Envelope, and the KLD for Dual-BRanch SSC, FOUR-BRANCH SEC, AND FOUR-BRANCH SECps IN THE LOS AND NLOS SCENARIOS FOR DIFFERENT SwITCHING THRESHOLDS

\begin{tabular}{|c|c|c|c|c|c|c|c|c|c|c|c|c|c|c|c|}
\hline \multirow{2}{*}{$\begin{array}{c}\text { Threshold } \\
\text { (dBm) }\end{array}$} & \multicolumn{5}{|c|}{ Dual-Branch SSC } & \multicolumn{5}{|c|}{ Four-Branch SEC } & \multicolumn{5}{|c|}{ Four-Branch SECps } \\
\hline & $\bar{m}$ & $\bar{\Omega}$ & $\bar{P}(\mathrm{dBm})$ & KLD & $\sigma$ & $\bar{m}$ & $\bar{\Omega}$ & $\bar{P}(\mathrm{dBm})$ & KLD & $\sigma$ & $\bar{m}$ & $\bar{\Omega}$ & $\bar{P}(\mathrm{dBm})$ & KLD & $\sigma$ \\
\hline & \multicolumn{15}{|c|}{ LOS Walking Scenario } \\
\hline-80 & 0.90 & 0.92 & -65.08 & 0.02 & 0.86 & 0.94 & 0.93 & -66.44 & 0.06 & 0.80 & 0.94 & 0.93 & -66.44 & 0.06 & 0.80 \\
\hline-60 & 0.74 & 0.71 & -63.28 & 0.03 & 0.84 & 0.78 & 0.61 & -63.28 & 0.11 & 0.75 & 0.76 & 0.58 & -63.08 & 0.07 & 0.78 \\
\hline \multirow[t]{2}{*}{-40} & 0.88 & 0.88 & -65.56 & 0.02 & 0.86 & 0.85 & 0.85 & -67.37 & 0.04 & 0.84 & 0.53 & 0.36 & -61.13 & 0.05 & 0.81 \\
\hline & \multicolumn{15}{|c|}{ NLOS Walking Scenario } \\
\hline-80 & 0.92 & 0.86 & -74.25 & 0.04 & 0.83 & 0.94 & 0.84 & -75.07 & 0.08 & 0.78 & 0.94 & 0.83 & -75.07 & 0.09 & 0.76 \\
\hline-60 & 0.73 & 0.63 & -71.73 & 0.06 & 0.79 & 0.60 & 0.46 & -70.55 & 0.10 & 0.76 & 0.55 & 0.41 & -69.75 & 0.09 & 0.76 \\
\hline-40 & 0.84 & 0.85 & -74.23 & 0.04 & 0.83 & 0.92 & 0.89 & -75.25 & 0.02 & 0.87 & 0.58 & 0.37 & -68.99 & 0.11 & 0.74 \\
\hline
\end{tabular}

as a PSC scheme, monitoring the signal level of all the branches continuously and simultaneously.

\section{Modeling of the Fading Observed at the Combiner Output}

The modeling of the fading characteristics at the output of the hypothetical combiners considered in this communication was performed using the models presented in Section II. The Nakagami $m$ and $\Omega$ parameters of the input signals were estimated using a nonlinear least squares routine programmed in MATLAB to fit (4), (6), and (8) to the measured data. To allow a direct comparison between the fading signals, the root mean square (rms) signal level was removed from the output envelope. As an example of the results of the model fitting, the pdfs of four-branch SEC and SECps with three different switching thresholds for the LOS walking scenario during the second trial are shown in Fig. 4. The pdfs of the four-branch SEC and SECps were in very good agreement ${ }^{3}$ with the measured data confirming the validity of the modeling approach utilized here.

${ }^{3}$ As a measure of the goodness-of-fit, the Kullback-Leibler divergence (KLD) [17] between the empirical and theoretical pdfs was calculated and is shown in Table II using KLD $=\int_{-\infty}^{\infty} f_{1}(x) \ln \left(f_{1}(x) / f_{2}(x)\right) d x$, where, in general, $f_{1}(x)$ and $f_{2}(x)$ denote the true and test pdfs, respectively. For the case when $f_{1}(x)$ is a Gaussian pdf with zero-mean and unit variance and $f_{2}(x)$ is a Gaussian pdf with zero-mean and variance $\sigma^{2}$, then KLD $=$ $0.5\left(\sigma^{-2}-1\right)+\ln (\sigma)$.
To allow the reader to be produce their own simulated data based on the empirical data reported here, Table II shows the mean parameter estimates averaged over all the trials for dual-branch SSC, four-branch SEC, and four-branch SECps in the LOS and NLOS walking scenarios with three different switching thresholds. Table II also shows the numerical values of the KLD alongside the corresponding estimated the standard deviation, $\sigma$, of a zero-mean and $\sigma^{2}$ variance Gaussian pdf that is used to approximate a zero-mean, unit variance Gaussian pdf. It was clear, with the exception of SECps, that the estimated $\Omega$ parameters (scale parameter) for the medium-switching threshold $(-60 \mathrm{dBm})$, were smaller than those obtained for the low and high switching thresholds. The subsequent narrowing effect on the output envelopes can be observed in Fig. 4(b) and (e). When compared with the pdfs for the low switching threshold shown in Fig. 4(a) and (d), it was obvious that they had a lower number of signal observations at low levels, suggesting that improvements in the received signal were achieved. As shown in Table II, this observation is also supported by the mean signal power of the output envelopes $(\bar{P})$ for the medium switching threshold, which were greater than the low switching threshold.

When the switching threshold is high $(-40 \mathrm{dBm})$, the four-branch SEC switched to another branch almost every single time slot, even if the currently selected branch had the highest signal level. This unnecessary path switching causes considerable signal fluctuation. 
However, for the above-mentioned reasons, the four-branch SECps scheme does not switch every single time slot. Therefore, four-branch SEC [Fig. 4(c)] had an empirical pdf with a greater spread and a larger number of signal observations at low signal levels than the four-branch SECps [Fig. 4(f)].

\section{CONCLUSION}

The potential improvement in the received signal for off-body communications at $5.8 \mathrm{GHz}$ using dual-branch SSC, dual- and four-branch SEC, and dual- and four-branch SECps has been evaluated in terms of their DG and compared with PSC. Among these switched diversity schemes, SECps provides a better performance than both the SSC and the SEC. It has been observed that up to $7.9 \mathrm{~dB}$ of DG can be achieved when using four-branch SECps. The impact of different switching thresholds has also been investigated and the importance of selecting the optimum switching threshold has been emphasized in this communication. It has also been shown that, for the scenarios considered in this communication, an SECps scheme provided almost the same performance as a PSC scheme when the optimum switching threshold was chosen. Finally, an analysis of the output envelope of the dual-branch SSC, four-branch SEC, and SECps operating in i.i.d Nakagami- $m$ fading channels has been presented. For all the measurements, the estimated KLD values were always $<0.12$, while the corresponding estimated $\sigma$ values of the Gaussian pdf with zero-mean and $\sigma^{2}$ variance were always greater than 0.73 . These results indicate that the theoretical models provided an adequate fit to the measured off-body channel data.

\section{REFERENCES}

[1] W. C. Jakes, Microwave Mobile Communications. New York, NY, USA Wiley, 1974.

[2] J. Boutros and E. Viterbo, "Signal space diversity: A powerand bandwidth-efficient diversity technique for the Rayleigh fading channel," IEEE Trans. Inf. Theory, vol. 44, no. 4, pp. 1453-1467, Jul. 1998.

[3] M. D. Yacoub, Foundations of Mobile Radio Engineering. Boca Raton, FL, USA: CRC Press, 1993.
[4] M. K. Simon and M.-S. Alouini, Digital Communication Over Fading Channels, 2nd ed. New York, NY, USA: Wiley, 2005.

[5] A. B. Sediq and H. Yanikomeroglu, "Performance analysis of soft-bit maximal ratio combining in cooperative relay networks," IEEE Trans. Wireless Commun., vol. 8, no. 10, pp. 4934-4939, Oct. 2009.

[6] T. Liang and D. B. Smith, "Energy-efficient, reliable wireless body area networks: Cooperative diversity switched combining with transmit power control," Electron. Lett., vol. 50, no. 22, pp. 1641-1643, Oct. 2014.

[7] Q. H. Abbasi, M. M. Khan, A. Alomainy, and Y. Hao, "Diversity antenna techniques for enhanced ultra wideband body-centric communications," in Proc. IEEE APSURSI, Washington, DC, USA, Jul. 2011, pp. 1323-1326.

[8] S. L. Cotton and W. G. Scanlon, "Measurements, modeling and simulation of the off-body radio channel for the implementation of bodyworn antenna diversity at $868 \mathrm{MHz}, "$ IEEE Trans. Antennas Propag., vol. 57, no. 12, pp. 3951-3961, Dec. 2009.

[9] I. Khan, P. S. Hall, A. A. Serra, A. R. Guraliuc, and P. Nepa, "Diversity performance analysis for on-body communication channels at 2.45 GHz," IEEE Trans. Antennas Propag., vol. 57, no. 4, pp. 956-963, Apr. 2009.

[10] Q. H. Abbasi et al., "Ultra wideband antenna diversity characterisation for off-body communications in an indoor environment," IET Microw., Antennas Propag., vol. 8, no. 14, pp. 1161-1169, 2014.

[11] H.-C. Yang and M.-S. Alouini, "Performance analysis of multibranch switched diversity systems," IEEE Trans. Commun., vol. 51, no. 5, pp. 782-794, May 2003.

[12] R. Rosini and R. D'Errico, "Off-body channel modelling at $2.45 \mathrm{GHz}$ for two different antennas," in Proc. IEEE EuCAP, Prague, Czech Republic, Mar. 2012, pp. 3378-3382.

[13] W. Braun and U. Dersch, "A physical mobile radio channel model," IEEE Trans. Veh. Technol., vol. 40, no. 2, pp. 472-482, May 1991.

[14] M. D. Yacoub, C. R. C. M. da Silva, and J. E. V. Bautista, "Second-order statistics for diversity-combining techniques in Nakagami-fading channels," IEEE Trans. Veh. Technol., vol. 50, no. 6, pp. 1464-1470, Nov. 2001.

[15] H.-C. Yang and M.-S. Alouini, "Improving the performance of switched diversity with post-examining selection," IEEE Trans. Wireless Commun., vol. 5, no. 1, pp. 67-71, Jan. 2006.

[16] A. M. D. Turkmani, A. Arowojolu, P. A. Jefford, and C. J. Kellett, "An experimental evaluation of the performance of two-branch space and polarization diversity schemes at $1800 \mathrm{MHz}$," IEEE Trans. Veh. Technol., vol. 44, no. 2, pp. 318-326, May 1995.

[17] S. Kullback, Information Theory and Statistics. Chelmsford, MA, USA: Courier, 1997. 